ARTICLE

\title{
Accumulation of heme biosynthetic intermediates contributes to the antibacterial action of the metalloid tellurite
}

Eduardo H. Morales ${ }^{1, \star}$, Camilo A. Pinto ${ }^{1, \star}$, Roberto Luraschi ${ }^{1}$, Claudia M. Muñoz-Villagrán ${ }^{1}$, Fabián A. Cornejo ${ }^{1}$, Scott W. Simpkins ${ }^{2}$, Justin Nelson², Felipe A. Arenas ${ }^{1}$, Jeff S. Piotrowski ${ }^{3}$, Chad L. Myers ${ }^{2,4}$, Hirotada Mori ${ }^{5}$

\& Claudio C. Vásquez ${ }^{1}$

The metalloid tellurite is highly toxic to microorganisms. Several mechanisms of action have been proposed, including thiol depletion and generation of hydrogen peroxide and superoxide, but none of them can fully explain its toxicity. Here we use a combination of directed evolution and chemical and biochemical approaches to demonstrate that tellurite inhibits heme biosynthesis, leading to the accumulation of intermediates of this pathway and hydroxyl radical. Unexpectedly, the development of tellurite resistance is accompanied by increased susceptibility to hydrogen peroxide. Furthermore, we show that the heme precursor 5-aminolevulinic acid, which is used as an antimicrobial agent in photodynamic therapy, potentiates tellurite toxicity. Our results define a mechanism of tellurite toxicity and warrant further research on the potential use of the combination of tellurite and 5 -aminolevulinic acid in antimicrobial therapy.

\footnotetext{
${ }^{1}$ Facultad de Química y Biología, Universidad de Santiago de Chile, Santiago 9170022, Chile. ${ }^{2}$ University of Minnesota-Twin Cities, Bioinformatics and Computational Biology, Minneapolis, Minnesota 55455, USA. ${ }^{3}$ Yumanity Therapeutics, Cambridge, Massachusetts 02139, USA. ${ }^{4}$ University of MinnesotaTwin Cities, Department of Computer Science and Engineering, Minneapolis, Minnesota 55455, USA. ${ }^{5}$ Graduate School of Biological Sciences, Nara Institute of Science and Technology, Ikoma 630-0101, Japan. * These authors contributed equally to this work. Correspondence and requests for materials should be addressed to H.M. (hmori@gtc.naist.jp) or to C.C.V. (email: claudio.vasquez@usach.cl).
} 
F or over 50 years, the oxyanion tellurite $\left(\mathrm{TeO}_{3}{ }^{2-}\right)$ has been known as a potent antibacterial ${ }^{1-3}$, ten times more toxic than mercury $\left(\mathrm{Hg}^{2+}\right)$ and several orders of magnitude more than other metals ${ }^{4}$. However, to date, the molecular basis of tellurite toxicity and resistance remains unclear.

Genetic determinants for tellurite resistance have been identified in both plasmids and the bacterial chromosome, including the ter, $k i l A$, teh $A B$, telAB and ars operons ${ }^{5-10}$, although the mechanism by which they act is unknown. Studies have suggested that enzymes of central metabolism are required for tellurite resistance ${ }^{11}$. When expressed in $E$. coli, the products of the ars and $\operatorname{teh} A B$ operons do not increase tellurite efflux or alter its uptake ${ }^{12}$, ruling out some potential resistance mechanisms. Recent studies identified several proteins that interact with TerC in E. coli ${ }^{13}$, which could improve the understanding on how TerC mediates tellurite resistance.

The current hypothesis of tellurite toxicity in bacteria is based on observations that tellurite depletes thiols ${ }^{14}$, which alters the reduction potential of the cell ${ }^{15}$, and elicits the production of reactive oxygen species (ROS) and ROS-detoxifying enzymes ${ }^{16,17}$. This hypothesis proposes that the main causes of tellurite toxicity is its oxidant nature ${ }^{11}$. Proteomic and transcriptomic studies in E. coli that identified enzymes required for ROS detoxification among the highest expressed after tellurite exposure support this hypothesis $^{18,19}$.

However, despite the evidence suggesting a role for ROS detoxification and thiols in tellurite resistance, some studies have challenged this idea. Inactivating GshA or TrxA in E. coli increased tellurite resistance in the presence of selenite ${ }^{20}$, suggesting that higher levels of glutathione and thioredoxin might be detrimental to tellurite resistance. Additionally, there is no definitive evidence linking metal toxicity to ROS production. For example, selenite, which is less toxic than tellurite by several orders of magnitude ${ }^{4}$, elicits higher ROS production than tellurite in $E$. coli ${ }^{20}$. Also, tellurite is still toxic under anaerobic conditions $^{21}$. Further, organisms that are ROS-resistant like Deinococcus radiodurans show only modest tellurite resistance ${ }^{15}$.

A major hurdle towards identifying tellurite resistance mechanisms and its toxicity has been the lack of truly resistant organisms for which genetic tools are available. While several studies characterized highly tellurite-resistant organisms, the genetic determinants and mechanisms of tellurite resistance were not identified ${ }^{22,23}$. To date, studies addressing this issue have been conducted with organisms that show modest or no tellurite resistance, making it difficult to identify the cellular target(s) and the true resistance mechanisms. To overcome this, in the present work, we developed an unbiased, genome-wide approach to understand tellurite toxicity and resistance. Using directed evolution and chemical genomics independently, we identified the heme biosynthetic pathway as a tellurite target. We confirmed that tellurite elicits the accumulation of protoporphyrin IX, which was correlated with iron depletion and cell death. Tellurite resistance required limiting substrate availability for the last steps of heme biosynthesis, where protoporphyrin IX (from here on proto IX) is synthesized. Increased hydrogen peroxide $\left(\mathrm{H}_{2} \mathrm{O}_{2}\right)$ or superoxide $\left(\mathrm{O}_{2}^{\bullet-}\right)$ detoxification or resistance was not required for tellurite resistance. In fact, tellurite-resistant strains were highly sensitive to $\mathrm{H}_{2} \mathrm{O}_{2}$, indicating that tellurite, $\mathrm{H}_{2} \mathrm{O}_{2}$ and $\mathrm{O}_{2}^{--}$ resistance are not directly correlated. Finally, we discovered that micromolar concentrations of the heme biosynthetic precursor 5-aminolevulinic acid (ALA) potentiate tellurite toxicity under both aerobic and anaerobic conditions. Interestingly, ALA is widely used as a prodrug in photodynamic therapy (PDT) for the control of bacteria, parasites, fungi and so on ${ }^{24}$, suggesting that the combined use of tellurite and ALA could further improve the efficacy of PDT and, perhaps, be applied in other biocontrol therapies.

\section{Results}

Limiting ALA availability increases tellurite resistance. To understand the mode of action and cellular targets of tellurite, we performed directed evolution experiments to isolate spontaneous E. coli-resistant mutants that showed robust growth in lysogeny broth (LB) medium containing increasing concentrations of tellurite up to $400 \mu \mathrm{g} \mathrm{ml}^{-1}$ (Fig. 1a). We isolated clonal strains from the evolved cultures by plating aliquots on LB plates and selecting isolated colonies from each day for further study. Individual colonies were tested for resistance following growth in LB medium (Fig. 1b and Supplementary Fig. 1a) containing
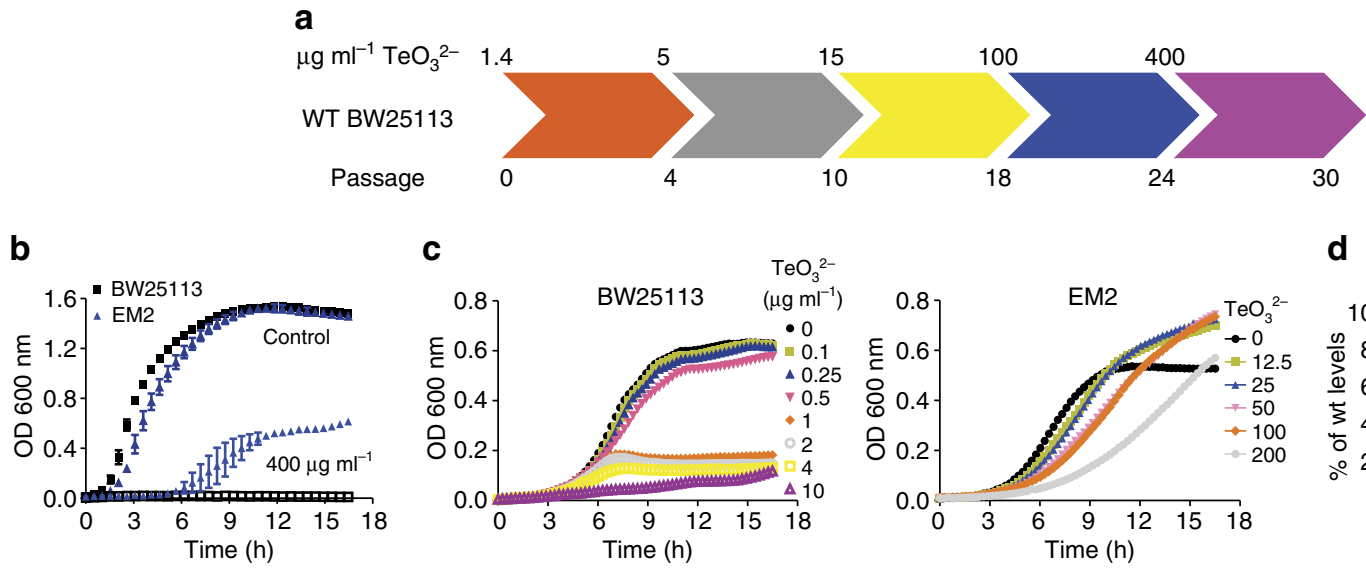

d

Figure 1 | Selection of mutants with increased tellurite resistance. (a) Summary of directed evolution experiment in LB medium. The number of serial passages (one passage $=$ growth for $24 \mathrm{~h}$ ) and the concentration of tellurite used is indicated. Strain EM2 was isolated at passage 26 . Representative growth curves of strains BW25113 and EM2 in (b) LB or (c) M9 medium in the presence of the indicated tellurite concentrations. Values represent the mean of three biological replicates. In (c) s.d. was removed for clarity (growth curves with s.d. are shown in Supplementary Fig. 2). (d) The indicated strains were grown in M9 medium and the heme and ALA content was measured in cell extracts. The level of heme or ALA in strain EM2, EM42 and EM43 were compared to strain BW25113, which was arbitrarily set to 100\%. Values represent the mean of three biological replicates. Error bars represent s.d. $\mathrm{TeO}_{3}^{2}-$ : tellurite ${ }^{\star \star} P<0.001 ;{ }^{\star \star \star} P<0.0001$ as determined by $t$-test. 
increasing concentrations of tellurite. Three strains with low $\left(15 \mu \mathrm{g} \mathrm{ml}^{-1}\right.$, strain EM40), intermediate $\left(100 \mu \mathrm{g} \mathrm{ml}^{-1}\right.$, strain EM 41) or high resistance $\left(400 \mu \mathrm{g} \mathrm{ml}^{-1}\right.$, strain EM2) were selected for genomic sequencing (Supplementary Table 1) together with the ancestral strain (BW25113).

Sequencing of the evolved strains revealed that mutations accumulated sequentially (Supplementary Table 1) and that they additively increased tellurite resistance (Fig. $1 \mathrm{~b}$ and Supplementary Fig. 1a). We selected strain EM2 for detailed study because it tolerated high tellurite concentrations in both LB (up to $400 \mu \mathrm{g} \mathrm{ml}^{-1}$; Fig. 1b) and M9 $0.2 \%$ glucose medium (up to $200 \mathrm{\mu g} \mathrm{ml}^{-1}$; Fig. 1c and Supplementary Fig. 2), levels comparable to other resistant organisms ${ }^{25}$. Unless otherwise indicated, all subsequent experiments were conducted in M9 $0.2 \%$ glucose medium (from here after referred as M9) to avoid possible chelation of tellurite by components present in LB medium, which could mask the effects of tellurite, as shown to occur with other metals ${ }^{26}$. Mutations found in strain EM2 (Supplementary Table 1) affected genes encoding proteins of pathways including transcription $(r p o D)$, response to membrane stress $(p s p F)$, synthesis of ALA (hemA and hemL) and the hypothetical protein YigE (Table 1 and Supplementary Table 1). Resistance conferred by the mutations in $r p o D$ and $p s p F$ likely act by inducing transcriptional reprogramming and by ameliorating the effect of tellurite on the $E$. coli cell membrane, respectively $y^{27,28}$. The role of the hypothetical protein YigE in tellurite resistance remains unclear, and further studies are being conducted to understand its role. The other two mutations conferring tellurite resistance are directly related to heme biosynthesis; hemA and hemL encode glutamyl tRNA reductase and glutamate semialdehyde 2,1-aminomutase, respectively, which catalyse the synthesis of ALA, the precursor of the pathway.

To determine the individual contributions of the observed mutations on tellurite resistance, we restored each mutation to the wild-type allele in strain EM2. Restoring the hemA mutation in strain EM2 with the wild-type allele (strain EM42) strongly reduced tellurite resistance and even impaired growth in the absence of any stress, as reflected by a longer lag phase and decreased growth. Conversely, restoring the wild-type hemL allele (strain EM44) in strain EM2 did not decrease tellurite resistance (Supplementary Fig. 1b), suggesting that the activity of the enzyme was not affected, consistent with a conservative aminoacid replacement. Introducing the hemA[31E] allele in an isogenic wild-type strain (strain EM43) was not sufficient to restore resistance of strain EM2 (Supplementary Fig. 1b), indicating that the genetic background is important for the observed tellurite resistance. Individually restoring the $p s p F$ or $r p o D$ mutations in strain EM2 (strains EM70 and EM64, respectively) decreased tellurite resistance by twofold as compared with strain EM2 (Supplementary Fig. 1b); however, restoring them together (strain EM71) had an additive effect and reduced growth under all the tellurite concentrations tested (Supplementary Fig. 1b). Together, our results indicate that the hemA mutation in strain EM2 shows a dominant effect on the increased tellurite resistance and suggests that altering the levels of ALA and/or heme is required for this process. It can be inferred that the mutation of hemA in strain EM2 did not cause a loss of function, as a null mutant requires the supplementation of exogenous ALA for growth and cannot synthesize heme ${ }^{29}$.

To test if the mutation of hemA in strain EM2 altered ALA and/or heme levels, these metabolites were quantified in strains BW25113, EM2, EM42 and EM43. The levels of ALA and heme were reduced in strains carrying mutant versions of HemA. In strains EM2 and EM43, the levels of ALA were 13\% and 55\% of those observed in strain BW25113, and heme was $40 \%$ and $68 \%$, respectively (Fig. 1d). Strains carrying wild-type alleles of $p s p F$, rpoD or hemL showed similar levels of heme than strain EM2, confirming that the hemA mutation caused the decreased levels of heme (Supplementary Fig. 1c). In summation, our results indicate that the mutation in hemA is responsible for the decreased levels of ALA and heme, which in turn increased tellurite resistance.

Chemical genomic analysis of tellurite toxicity. As a complementary approach to understand tellurite toxicity, we used chemical genomic profiling of E. coli ${ }^{30}$ to identify deletions that are responsive to tellurite. We challenged a pooled collection of $\sim 6,000$ deletion mutants with either tellurite or a solvent control (dimethylsulfoxide (DMSO)) in LB medium. Sequencing of strain-specific barcodes allowed us to determine the fitness of each mutant strain in the presence of tellurite relative to the solvent control (Fig. 2a and Supplementary Data 1).

Deletion mutants for 377 and 130 genes showed a significant threefold increase or decrease in fitness after tellurite exposure (Fig. 2a and Supplementary Data 1), respectively. Among the top deletion mutants sensitive to tellurite, we detected significant enrichment $(P<0.001$, hypergeometrical test) for genes involved in the Kyoto Encyclopedia of Genes and Genomes pathway of flagellar assembly (eco02040). Unexpectedly, no deletion mutants annotated to degradation and response to $\mathrm{H}_{2} \mathrm{O}_{2}$ or $\mathrm{O}_{2}^{--}$, iron sulfur cluster assembly, or glutathione metabolism, were among the top sensitive strains (Fig. 2a and Supplementary Data 1). Supporting these results, we found that catalase activity was

Table 1 | E. coli strains used in this study.

Strain

BW25113

EM2

$\Delta$ hemN

hemN $\mathrm{N}^{+}$

$\Delta$ hemF

EM40

EM41

EM42

EM43

EM44

EM53

EM64

EM70

EM71
Relevant genotype

lacla rrnBT14 $\Delta$ lacZWJ16 hsdR514 $\triangle$ araBADAH33, $\Delta$ rhaBADLD78

BW25113 yigE[A22V] rpoD[R603S] pspF[L52H] hemA[A31E] hemL[A118V]

BW25113 $\Delta$ hemN::aph

$\Delta$ hem $N$ complemented in cis

BW25113 $\Delta$ hemF::aph

BW25113 yigE[A22V]

BW25113 yigE[A22V] rpoD[R603S]

BW25113 hemA[A31E]

EM2 hemA wild type

EM2 hemL wild type

BW25113 yigE[A22V] rpoD[R603S]

EM2 rpoD wild type

EM2 pspF wild type

EM2 rpoD pspF wild type

\section{Source}

Keio collection ${ }^{31}$

This study

Keio collection ${ }^{31}$

This study

Keio collection ${ }^{31}$

This study

This study

This study

This study

This study

This study

This study

This study

This study 
a

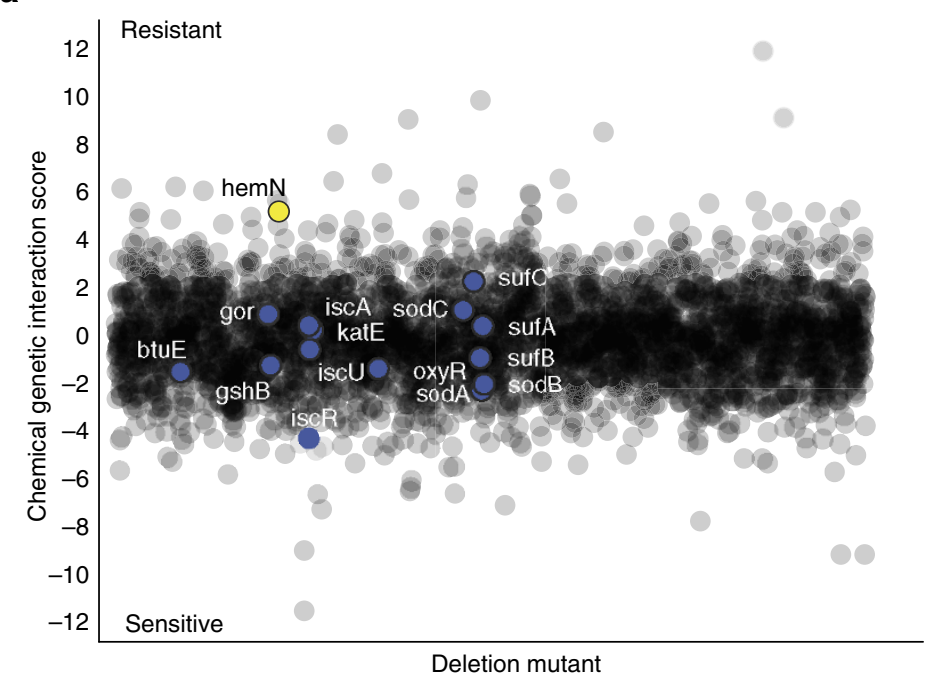

C

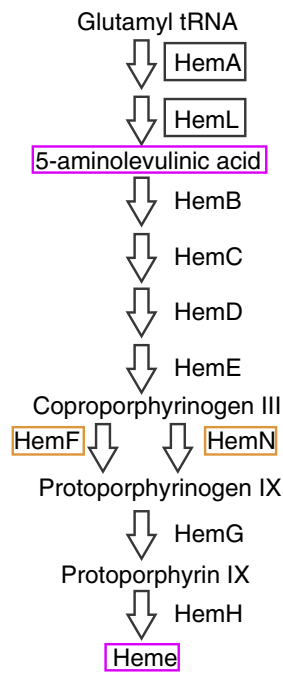

b
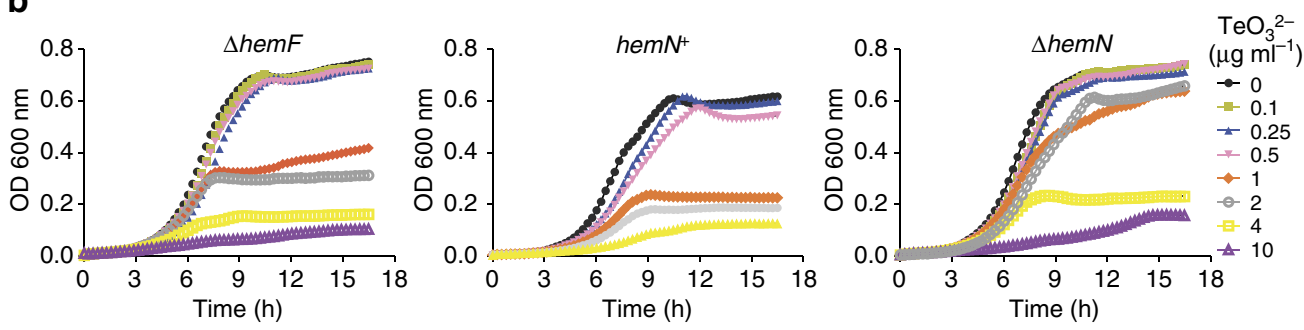

Figure 2 | Chemical genomics of tellurite. (a) Treatment of the E. coli barcoded deletion collection with tellurite. Positive or negative chemical genetic interaction scores represent increased or decreased tellurite resistance, respectively. Selected deletion mutants of genes encoding proteins required for heme biosynthesis (hemN; yellow circle), glutathione metabolism (gor, gshB), ROS resistance (btuE, oxyR, sodA, $\operatorname{sodB} \operatorname{sodC}$, katE) and iron-sulfur cluster assembly (isc $A$, iscR, isc $U$, sufA, sufB, sufC) are indicated. (b) Representative growth curves of strains $\Delta$ hemF, hemN $N^{+}$and $\Delta$ hemN in $M 9$ medium in the presence of the indicated tellurite concentrations. Values represent the mean of three biological replicates. s.d. was removed for clarity (growth curves with s.d. are shown in Supplementary Fig. 4). (c) Schematic representation of heme biosynthetic pathway in E. coli BW25113. Selected proteins encoded by genes that when inactivated (orange box) or mutated (black box) increased tellurite resistance or heme biosynthetic intermediates (magenta box) that showed altered levels in strain $\mathrm{EM} 2$ are highlighted. $\mathrm{TeO}_{3}^{2-}$ : tellurite.

fivefold lower in strain EM2 than in strain BW25113, and did not change with either $\mathrm{H}_{2} \mathrm{O}_{2}$ or tellurite exposure. Further, superoxide dismutase activity, tellurite reductase (TR) activity and thiol levels were similar between both strains (Supplementary Fig. 3a,d,e). Consistent with the lower levels of catalase activity, strain EM2 was more sensitive to $\mathrm{H}_{2} \mathrm{O}_{2}$ than strain BW25113 (Supplementary Fig. 3b,c). ALA supplementation restored catalase activity in strain EM2 to wild-type levels, indicating that the decreased activity was due to lower heme content (Supplementary Fig. 3a). Previously, it was proposed that $\mathrm{H}_{2} \mathrm{O}_{2}$ and $\mathrm{O}_{2}^{-}-$are key intermediates in tellurite toxicity, and that decreasing their levels and maintaining the pool of reduced glutathione are required for tellurite resistance ${ }^{14,17}$. However, our results provide evidence that there is no direct correlation between thiol levels, $\mathrm{H}_{2} \mathrm{O}_{2}, \mathrm{O}_{2}^{\bullet-}$ and tellurite resistance.

Among the top deletion mutants resistant to tellurite, we detected enrichment for genes involved in the gene ontology category of cofactor biosynthesis $(P<0.01$; gene ontology: 0051188; hypergeometrical test) and in the Kyoto Encyclopedia of Genes and Genomes pathway of porphyrin and chlorophyll metabolism $(P<0.05$; hypergeometrical test). In both cases, a deletion strain for a gene implicated in heme biosynthesis, hemN, was included (Fig. 2a and Supplementary Data 1). HemN is an oxygen-independent coproporphyrinogen III (copro III) dehydrogenase that is functional under both aerobic and anaerobic conditions ${ }^{31,32}$. It is not surprising that no other deletion strains for genes encoding enzymes of heme synthesis were found in the analysis, as most of these enzymes are essential for aerobic growth in the LB medium ${ }^{33}$. Nevertheless, because the genome of $E$. coli encodes two enzymes able to convert copro III to protoporphyrinogen IX that can be individually inactivated, namely $\mathrm{HemN}$ and $\mathrm{HemF}$, it allowed testing if deleting genes downstream of hemA also increased tellurite resistance. We confirmed the resistance of the individual $\Delta h e m F$ and $\Delta h e m N$ strains and found that they were able to grow in medium containing up to 2 and $4 \mu \mathrm{g} \mathrm{ml}^{-1}$ tellurite, respectively (Fig. $2 \mathrm{~b}$ and Supplementary Fig. 4). Complementation of strain $\Delta$ hemN (strain hem $N^{+}$) restored tellurite sensitivity to wild-type levels (Fig. 2b and Supplementary Fig. 4), confirming the observed result.

Taken together, the chemical genomic analysis and directed evolution approach indicate that limiting substrate availability for the last two steps of heme biosynthesis increased tellurite resistance (Fig. 2c). Based on these results, we speculated that tellurite might either interact with metabolites downstream of copro III or cause their accumulation, which could result in cell death.

Tellurite induces the accumulation of heme intermediates. Following the logic that tellurite exposure could lead to the accumulation of heme intermediates downstream of the reaction catalysed by HemN and HemF, we quantified the levels of 
copro III and proto IX in cells exposed to tellurite. Because protoporphyrinogen IX spontaneously oxidizes to proto IX on extraction 34 , the values presented represent the sum of both intermediates. In BW25113, the levels of proto IX increased 60 -fold after tellurite exposure (Fig. 3a,b) and cell viability decreased by 2,000-fold (Fig. 3c). Conversely, proto IX was undetectable in strains $\Delta h e m N$ and EM2, and only copro III was detected in strain $\Delta h e m N$, although the levels remained unchanged after tellurite exposure (Fig. 3a,b). As expected, tellurite did not affect the growth of strain EM2, and the viability of strain $\Delta$ hemN only decreased by 70 -fold (Fig. 3c), a lower reduction than for strain BW25113. Complementing strain $\Delta h e m N$ resulted in wild-type levels of porphyrins and increased tellurite sensitivity (Fig. 3a-c). No significant changes in the levels of intracellular tellurite were observed between the tested strains (Fig. 3d), ruling out that the differences in the accumulation of heme intermediates and in cell viability were caused by decreased exposure to the toxic compound.
ALA potentiates tellurite toxicity. An alternative approach to test if tellurite toxicity is caused by the accumulation of intermediates in heme biosynthesis is to increase the availability of substrates for the pathway. In E. coli, ALA, the precursor of the pathway, is transported by the dipeptide transport system encoded by the $d p p$ operon ${ }^{35}$ and can be used for this purpose. The rationale is that if strain EM2 acquired at least part of tellurite resistance by limiting ALA levels, then supplementing the growth medium with exogenous ALA should bypass the effect of the hemA mutation and decrease tellurite resistance. To compare directly the effect of ALA on the growth of strain BW25113 and EM2, the concentration of tellurite was increased for strain EM2 to levels $\left(400 \mu \mathrm{g} \mathrm{ml}^{-1}\right)$ that resulted in a similar decrease in cell viability than those observed for strain BW25113 (Fig. 3c). Sublethal concentrations of ALA $\left(25 \mu \mathrm{g} \mathrm{ml}^{-1}\right)$ strongly sensitized strain EM2 to tellurite and cell viability decreased to almost undetectable levels after $180 \mathrm{~min}$ of exposure (Fig. 4a). In contrast, EM 2 cells grown in the absence of ALA and exposed a
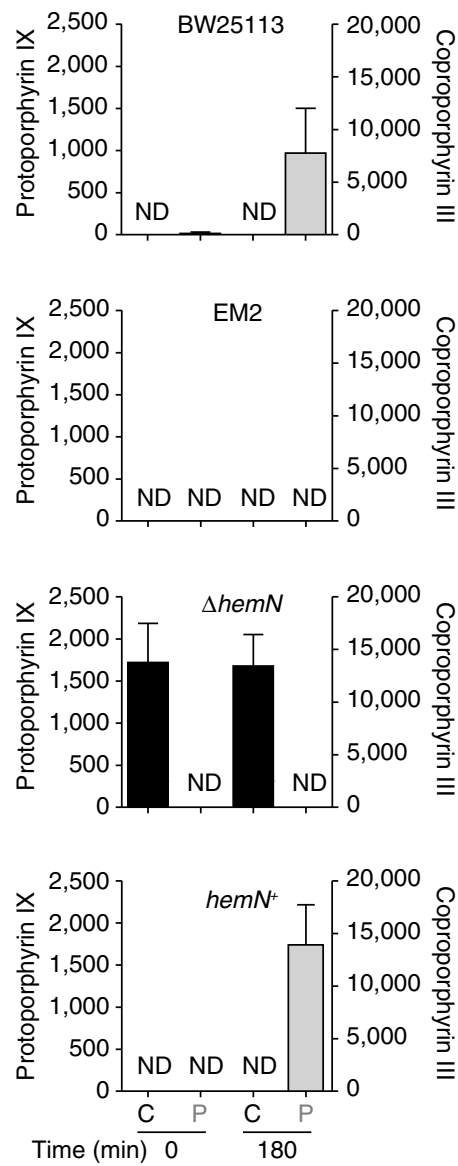

b
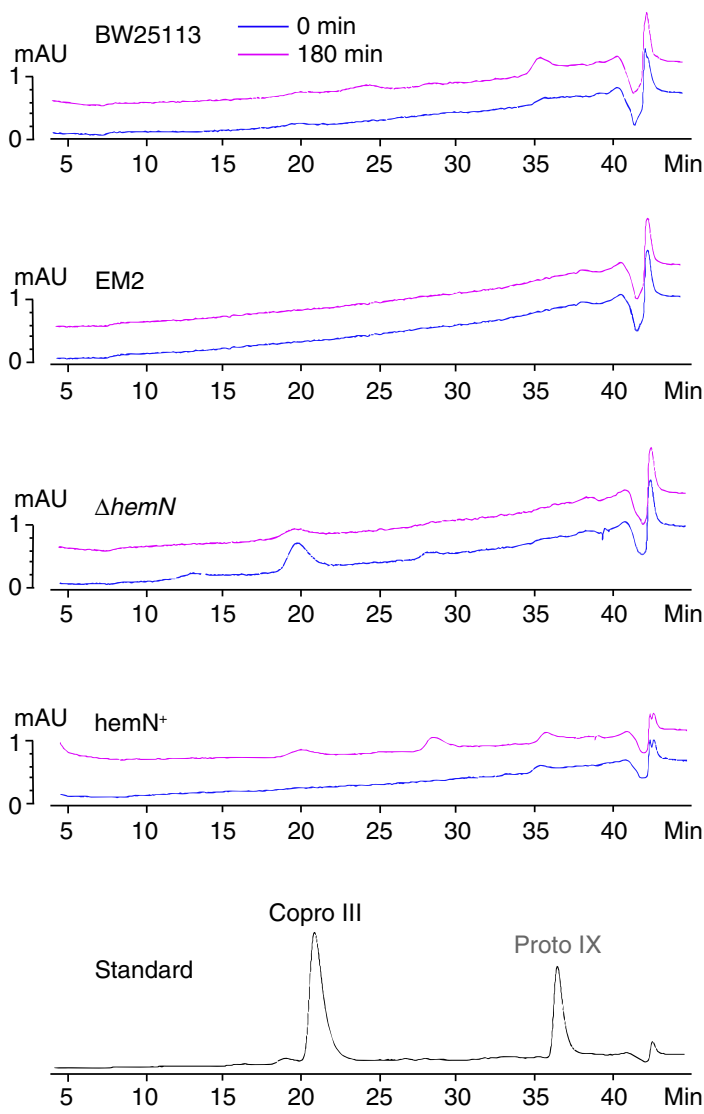

C
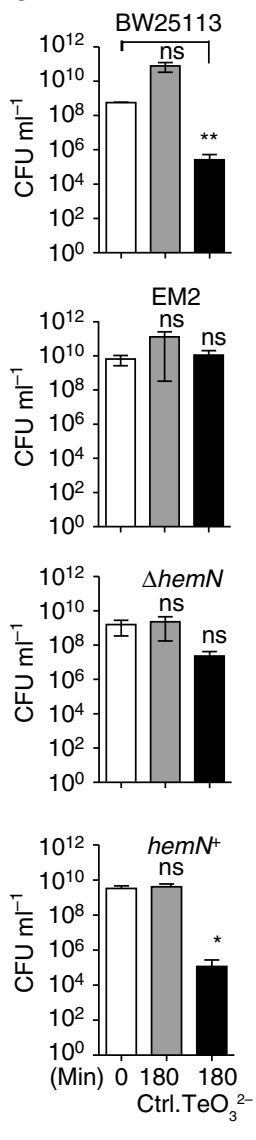

d

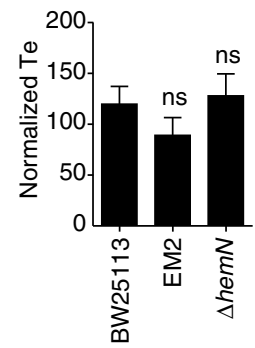

Figure 3 | Proto IX accumulates in tellurite exposed cells. (a) Strains BW25113, EM2, $\Delta$ hemN and hemN ${ }^{+}$were grown in M9 medium, challenged with tellurite $\left(25 \mu \mathrm{g} \mathrm{ml}^{-1}\right)$ and the porphyrin content of the cell was extracted and quantified by HPLC. In parallel, commercially available standards were quantified and used to identify each peak. Proto IX represents the sum of protoporphyrinogen IX and proto IX. Units for proto IX and copro III are nanomoles normalized by $\mathrm{mg}$ of protein. Time under the graph represents the time of porphyrin extraction with respect to tellurite exposure. $\mathrm{C}=$ coproporphyrin III (black bars); $\mathrm{P}=$ proto IX (gray bars). Values represent the mean of 3 biological replicates. Error bars represent s.d. ND = not detected. (b) Representative HPLC chromatograms of samples from each strain quantified in a. Blue ( 0 min) and magenta lines (180 min) in each chromatogram represent the time at which porphyrins were extracted with respect to tellurite addition. (c) CFU ml ${ }^{-1}$ were quantified from the same samples used for porphyrin extraction shown in a. Ctrl = untreated cells. Time under the graph represents the time at which CFU $\mathrm{ml}^{-1}$ were determined with respect to tellurite exposure. Values represent the mean of three biological replicates. Error bars represent s.d. ns, not significant; ${ }^{\star} P<0.05$; ${ }^{\star \star} P<0.001$ as determined by $t$-test. (d) ICP-OES analyses. Strains BW25113, EM2 and $\Delta$ hemN were grown in M9 medium, challenged with tellurite $\left(25 \mu \mathrm{g} \mathrm{ml}^{-1}\right)$ and intracellular tellurium was quantified by ICP-OES analysis after $180 \mathrm{~min}$. Units for normalized Te are $\mu \mathrm{g} \mathrm{ml} \mathrm{m}^{-1}$ of tellurium $\mathrm{g}$ of cell pellet $^{-1}$. Values represent the mean of three biological replicates. Error bars represent s.d. ns: not significant as determined by $t$-test. TeO ${ }^{2-}:$ tellurite. 
a

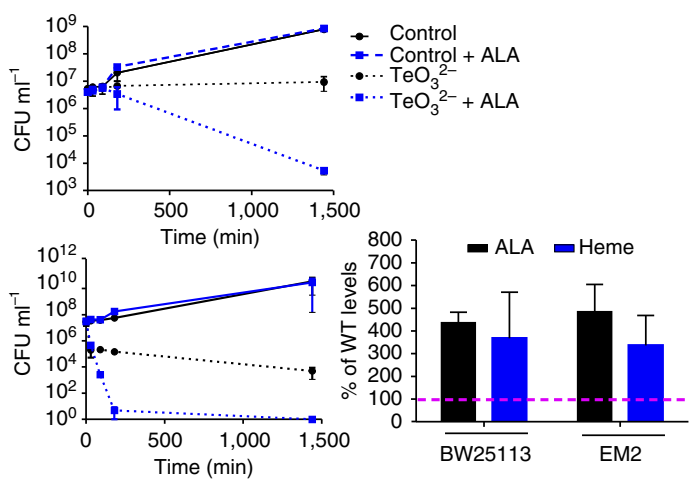

b

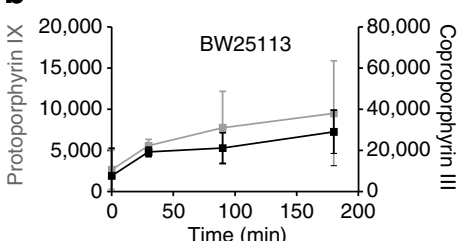

C

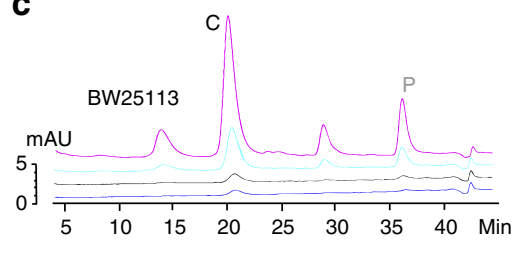

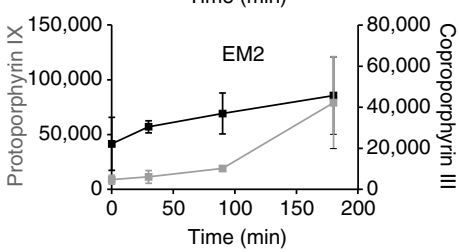

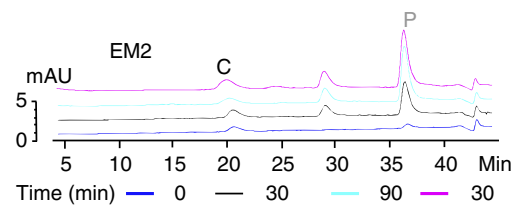

d
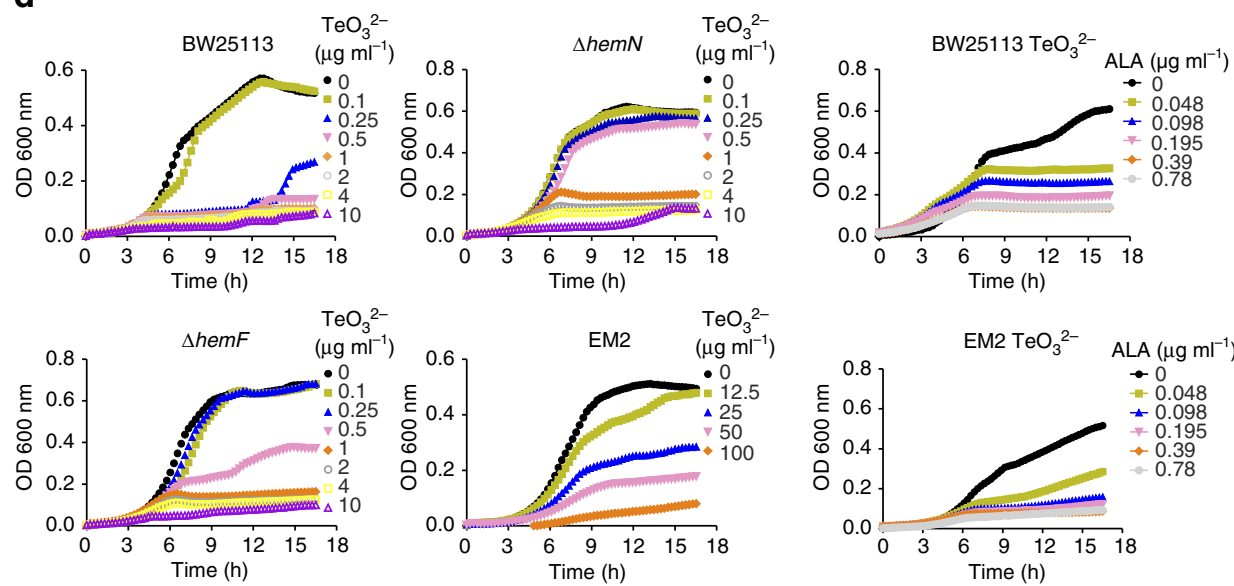

Figure 4 | ALA potentiates tellurite toxicity. (a) Strains BW25113 (upper panel) and EM2 (lower panel) were grown in M9 medium supplemented or not with $25 \mathrm{\mu g} \mathrm{ml}^{-1} \mathrm{ALA}$, challenged or not with tellurite and CFU ml ${ }^{-1}$ were quantified at the indicated time points. Strains BW25113 and EM2 were exposed to 10 and $400 \mu \mathrm{g} \mathrm{ml}^{-1}$ tellurite, respectively. Heme and ALA content were determined from cell extracts. Levels of heme and ALA in strains BW25113 and EM2 were compared with those of strain BW25113 grown without ALA (dotted magenta line, from Fig. 1d). Values represent the mean of three biological replicates. Error bars represent s.d. (b) Strains BW25113 and EM2 were grown in M9 medium supplemented with $25 \mu \mathrm{g} \mathrm{ml}{ }^{-1}$ ALA and challenged with tellurite (25 and $400 \mu \mathrm{g} \mathrm{ml}^{-1}$ for strains BW25113 and EM2, respectively) and the porphyrin content was extracted and quantified by HPLC at the indicated time points. Units for proto IX and coproporphyrin III are nanomoles normalized by $\mathrm{mg}$ of protein. Values represent the mean of three biological replicates. Error bars represent s.d. (c) Representative HPLC chromatograms of samples from each strain quantified in $\mathbf{b}$. The colour of each chromatogram (indicated at the bottom of the figure) represents the time at which porphyrins were extracted regarding to tellurite addition. $\mathrm{C}=$ coproporphyrin III;

$\mathrm{P}=$ protoporphyrin IX. (d) Representative growth curves of strains BW25113, EM2, $\Delta$ hemN and $\Delta$ hemF in M9 medium supplemented with $25 \mu \mathrm{g} \mathrm{ml}^{-1} \mathrm{ALA}^{-}$ in the presence of the indicated tellurite concentrations. Values represent the mean of three biological replicates. S.d. was removed for clarity (growth curves with s.d. are shown in Supplementary Fig. 5). (e) Representative growth curves of strains BW25113 and EM2 in M9 medium with tellurite (0.5 and $50 \mathrm{\mu g} \mathrm{ml}^{-1}$, respectively) supplemented with the indicated concentrations of ALA. Values represent the mean of three biological replicates. S.d. was removed for clarity. Growth curves with s.d. are shown in Supplementary Fig. 6). $\mathrm{TeO}_{3}^{2-}$ : tellurite.

to the same amount of tellurite remained viable even after $24 \mathrm{~h}$ (Fig. 4a). We observed a similar result when BW25113 was treated with tellurite. In the absence of ALA, colony-forming units (CFU) $\mathrm{ml}^{-1}$ were 1,700 -fold higher than in cells grown in the presence of ALA (Fig. 4a). In both strains, ALA was equally incorporated and reached levels $400 \%$ higher than in strain BW25113 grown in M9 medium and had no effect on cell viability. A similar increase in heme content was also observed in both strains (Fig. 4a).

The increased tellurite toxicity in the presence of ALA could be caused by an accumulation of intermediates in heme biosynthesis. Indeed, we observed increased levels of proto IX, copro III and other unidentified intermediates of heme biosynthesis in BW25113 and EM2 grown with ALA and exposed to tellurite (Fig. 4b,c). In strain EM2, proto IX and copro III accumulated over time and reached a 90 - and 2 -fold increase after $180 \mathrm{~min}$ of tellurite exposure, respectively. In strain BW25113, the levels of both metabolites increased 3.5-fold (Fig. 4b). Because BW25113 and EM2 were treated with different tellurite concentrations, we believe that the lower accumulation of proto IX and slower cell death observed in strain BW25113 as compared to strain EM2 are due to differences in the rate of tellurite incorporation. Supporting this view, the levels of intracellular tellurite were fourfold higher in strain EM2 treated with $400 \mu \mathrm{g} \mathrm{ml}^{-1}\left(763.98 \pm 131.1 \mu \mathrm{g} \mathrm{ml}^{-1}\right.$ of tellurium $g$ of cell pellet ${ }^{-1}$ ) than in strain BW25113 treated with $25 \mathrm{\mu g} \mathrm{ml}^{-1}\left(187.04 \pm 26.5 \mu \mathrm{g} \mathrm{ml}^{-1}\right.$ of tellurium $\mathrm{g}$ of cell pellet $\left.{ }^{-1}\right)$ at $180 \mathrm{~min}$. Additionally, we acknowledge that the levels of proto IX in cells grown with ALA are two- and eightfold higher in strains BW25113 and EM2 even before tellurite exposure (Fig. 4b) than in strain BW25113 treated with tellurite alone (Fig. 3a). This indicates that under the tested conditions, proto IX per se is not lethal to E. coli and might not be solely responsible for the observed death, but that the combination of proto IX and tellurite causes cell death.

Importantly, we revealed that ALA potentiates the toxic effects of tellurite and that this occurs in both a sensitive (BW25113) and a resistant strain (EM2) to tellurite (Fig. 4a). Yet, at this point, 


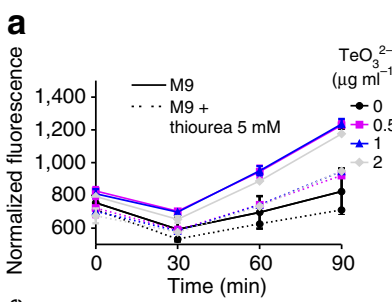

b
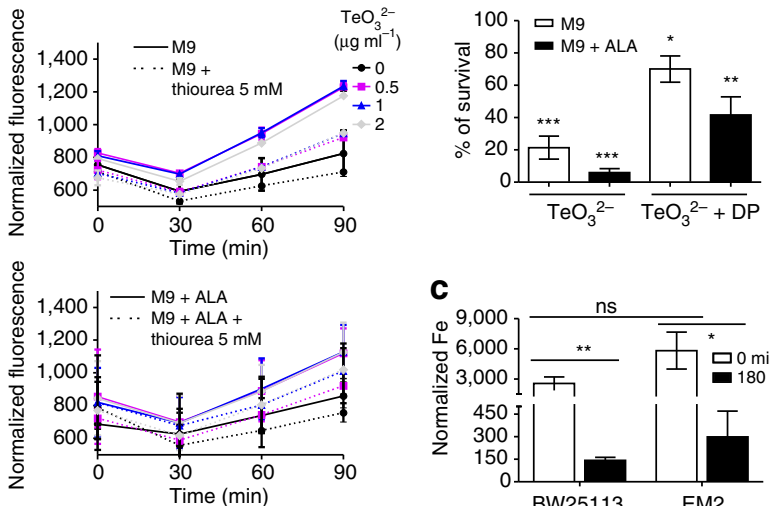

$\left(\begin{array}{ll}\mathrm{TeO}_{3}^{2-} \\ \left(\mu \mathrm{ml}^{-1}\right)\end{array}\right.$

$\rightarrow 0$

-0.5
+1

+1
-2

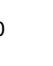

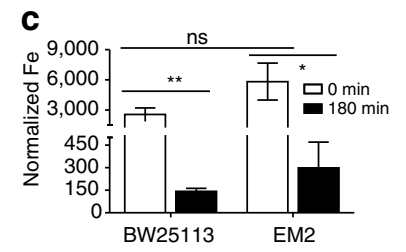

d
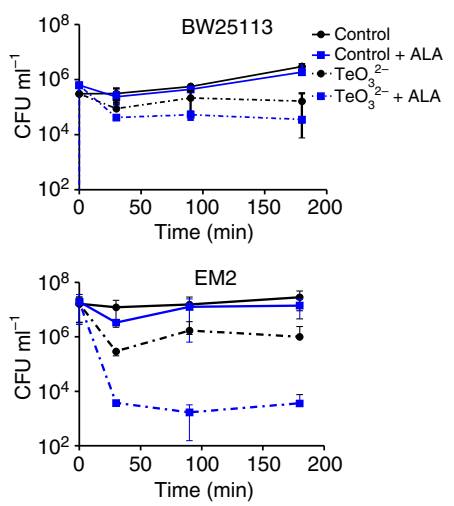

Figure 5 | Tellurite exposure causes iron depletion and $\mathbf{0} \mathbf{O H}$ generation. (a) Strain BW25113 was grown in M9 medium supplemented or not with $25 \mathrm{~g} \mathrm{ml}^{-1} \mathrm{ALA}$ or $5 \mathrm{mM}$ thiourea, and hydroxyl radical generation was determined at the indicated time points. Tellurite concentrations that were used are indicated. Values represent the mean of three independent experiments. Error bars represent s.d. (b) Strain BW25113 was grown in M9 medium

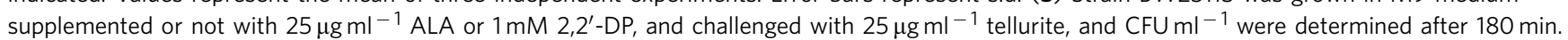
Per cent survival is with respect to the culture at time $0 \mathrm{~min}$. Values represent the mean of three biological replicates. Error bars represent s.d. ${ }^{\star} P<0.05$, ${ }^{\star \star} P<0.001$ and ${ }^{* \star \star} P<0.0001$ as determined by $t$-test. (c) ICP-OES analyses. Strain BW25113 was grown in M9 medium, challenged with tellurite $\left(25 \mathrm{~g} \mathrm{ml}^{-1}\right)$ and intracellular iron was quantified by ICP-OES analyses after $180 \mathrm{~min}$. Units for normalized Fe are $\mu \mathrm{g} \mathrm{ml}^{-1}$ of iron $\mathrm{g}$ of cell pellet ${ }^{-1}$. Values represent the mean of three biological replicates. Error bars represent s.d. ${ }^{\star} P<0.05$, ${ }^{\star \star} P<0.001$. NS, not significant as determined by $t$-test. (d) Overnight cultures of strains BW25113 and EM2 were grown anaerobically and inoculated into M9 medium supplemented or not with $25 \mu \mathrm{g} \mathrm{ml}{ }^{-1} \mathrm{ALA}$, and challenged or not with 50 or $400 \mathrm{\mu g} \mathrm{ml}^{-1}$ tellurite, respectively. At the indicated time points, $\mathrm{CFU} \mathrm{ml} \mathrm{m}^{-1}$ were quantified. Values represent the mean of three biological replicates. Error bars represent s.d. $\mathrm{TeO}_{3}^{2-}$ : tellurite.

the degree of this potentiation is unknown. To determine the minimal amount of tellurite required to inhibit growth in the presence of ALA, we challenged strains BW25113, DhemN, $\Delta h e m F$ and EM2 with decreasing amounts of tellurite in the presence of a fixed amount of ALA $\left(25 \mu \mathrm{g} \mathrm{ml}^{-1}\right)$. In all cases, the amount of tellurite required to inhibit growth was lower when ALA was present (compare Figs $1 \mathrm{c}$ and $2 \mathrm{~b}$ with Fig. $4 \mathrm{~d}$ and Supplementary Fig. 5). To further investigate the potentiation between these two compounds, we determined the minimum concentration of ALA that inhibited cell growth when combined with tellurite. As a proof of principle, these experiments were limited to strains BW25113 and EM2. We used a fixed amount of tellurite $\left(0.5\right.$ and $50 \mu \mathrm{g} \mathrm{ml}^{-1}$ for strain BW25113 and EM2, respectively) that allowed robust growth in the absence (Fig. 1b) but not in the presence of ALA (Fig. 4d), and varied the concentration of ALA. A concentration as low as $0.098 \mu \mathrm{g} \mathrm{ml}{ }^{-1}$ $(\sim 750 \mathrm{nM})$ ALA was enough to inhibit growth of strains BW25113 and EM2 (Fig. 4e and Supplementary Fig. 6). In summation, our results show that in E. coli, tellurite toxicity highly correlates with the accumulation of intermediates in heme biosynthesis, mainly proto IX, and that the addition of ALA further potentiates this effect.

Tellurite exposure leads to iron deficiency. Previous studies showed that under aerobic conditions, tellurite can damage the iron-sulfur cluster of some enzymes ${ }^{36}$, suggesting that the intracellular pool of free iron could be altered because of tellurite exposure. As porphyrins are a source of several $\operatorname{ROS}^{37,38}$ and also accumulate after tellurite exposure (Fig. 3e), we speculated that iron and $\mathrm{H}_{2} \mathrm{O}_{2}$ could react through the Fenton reaction, generating the highly oxidizing hydroxyl radical $\left(\bullet^{\circ} \mathrm{OH}\right)$, leading to cell death. Hydroxyl radical was detected in strain BW25113 challenged with tellurite and the levels increased over time. Supplementing the growth media with the ${ }^{\bullet} \mathrm{OH}$ quencher thiourea $^{39}$ resulted in fluorescence levels similar to those observed in untreated cells (Fig. 5a). A similar result was observed in cells grown with ALA. To test directly if iron played a role in tellurite toxicity, BW25113 cells were challenged with tellurite in the presence or absence of the iron chelator $2,2^{\prime}$-dipyridyl (DP). After $180 \mathrm{~min}$ of tellurite treatment, only $20 \%$ of cells remained viable, versus $70 \%$ in media supplemented with DP. A similar trend was observed in cells grown with ALA; only $6 \%$ of cells remained viable after tellurite treatment, versus $40 \%$ when DP was present (Fig. 5b). The previous experiments showed that decreasing iron levels increased tellurite resistance. Hence, we hypothesized that E. coli would respond to tellurite exposure by limiting iron availability. After $180 \mathrm{~min}$ of tellurite exposure, the levels of intracellular iron decreased 19-fold in strain BW25113, as compared to the starter culture. A similar decrease was observed in strain EM2 (Fig. 5c). Together, our experiments show that iron is harmful for the cell when exposed to tellurite, and that $E$. coli responds by drastically decreasing iron levels. However, this defense mechanism could be detrimental for the cell. Iron is a substrate for the last step of heme biosynthesis, catalysed by ferrochetalase, and limiting iron availability after tellurite exposure could be problematic and lead to proto IX accumulation, explaining, at least in part, the observed results.

These experiments show that ${ }^{\bullet} \mathrm{OH}$, but not $\mathrm{H}_{2} \mathrm{O}_{2}$ or $\mathrm{O}_{2}^{\bullet-}$, plays a role in tellurite toxicity. However, they do not rule out the possibility that proto IX, and perhaps other porphyrins, are also toxic. To directly test this, strains BW25113 and EM2 were grown under anaerobic conditions, where ROS are not generated, with or without $25 \mathrm{\mu g} \mathrm{ml}^{-1}$ ALA and challenged with 50 or $400 \mu \mathrm{g} \mathrm{ml}^{-1}$ tellurite, respectively. It was expected that if proto IX is toxic, then ALA should still potentiate tellurite toxicity. Strains BW25113 and EM2 remained viable when grown with or without ALA (Fig. 5d). However, as hypothesized, ALA strongly sensitized both strains to tellurite and CFU decreased $94.3 \%$ and $99.9 \%$, respectively, as compared to the starter cultures (Fig. 5d). At the very least, this means that tellurite has an acute mechanism of toxicity that is independent of ROS and is potentiated by ALA.

\section{Discussion}

Until now, the mechanism of tellurite resistance was unclear. To gain insights into this process, we developed an unbiased 
platform to identify novel mechanisms of tellurite resistance and intracellular targets. Resequencing a spontaneous E. coli-resistant strain predicted the heme biosynthetic pathway as a tellurite target (Supplementary Table 1 and Figs 1 and 2). Mutation of HemA at residue A31E in strain EM2 decreased ALA levels (Fig. 1d). Alignment of the amino-acid sequence of HemA from E. coli BW25113 and EM2 with that of other organisms showed that residue 31 is highly conserved and located at the catalytic N-terminal domain (Supplementary Fig. 7), based on the crystal structure of HemA from Methanopyrus kandleri ${ }^{40}$. Hence, replacing the small and nonpolar amino-acid alanine with a polar, charged amino acid with a bulky side chain like glutamic acid could affect the folding and stability of the domain and influence the surface involved in interactions with the substrate, affecting the production of ALA.

The other two mutations found in strain EM2 that increased tellurite resistance (Supplementary Fig. 1b) affected the genes encoding the housekeeping sigma factor RpoD and the transcription factor PspF. Mutation of RpoD residue R603 (Supplementary Table 1) has been previously isolated and selected as conferring increased fitness to ethanol stress, and is implicated in a reduction of transcriptional capacity ${ }^{27}$. Hence, it is predicted that the mutation of RpoD R603S has a pleiotropic effect and alters the transcriptional profile of strain EM2 as compared to strain BW25113. PspF is the transcriptional activator of the stress inducible $p s p$ operon $^{28}$, which is induced in conditions of membrane stress that most likely result in the impairment of the proton motif force. We hypothesize that mutation of PspF at residue L52 alters the expression of the $p s p$ operon, allowing the cell to cope more promptly and efficiently to the stress generated by tellurite on the cell membrane.

Screening a barcoded $E$. coli deletion collection revealed that deleting hem $N$, required for the conversion of copro III to protoporphyrinogen IX, increased tellurite resistance (Fig. 2a.b and Supplementary Fig. 4). For many years, HemF was considered the main enzyme catalysing the conversion of copro III to protoporphyrinogen IX in aerobiosis, although no studies have addressed the individual contribution of each enzyme in vivo. However, recent studies suggest that $\mathrm{HemN}$ is the housekeeping enzyme in this process ${ }^{34}$, because it is functional under oxic and anoxic conditions ${ }^{31,32}$ and that under aerobic conditions the levels of HemN are fourfold higher than those of $H_{e m F^{41}}$. This could explain why strain $\Delta$ hemN showed the greatest increase in tellurite resistance, as it would accumulate higher levels of copro III, limiting substrate availability for the last steps of heme biosynthesis.

Unexpectedly, tellurite resistance arose from limiting heme biosynthesis with the trade-off of decreasing $\mathrm{H}_{2} \mathrm{O}_{2}$ resistance and catalase activity. Further, increased superoxide detoxification was not required (Supplementary Fig. $3 \mathrm{a}-\mathrm{c}$ ), indicating that neither $\mathrm{H}_{2} \mathrm{O}_{2}$ nor $\mathrm{O}_{2}^{\bullet-}$ play a key role in tellurite toxicity. This is somehow counterintuitive, as tellurite alone or combined with ALA were more efficient at killing $E$. coli under aerobic conditions (Figs $4 \mathrm{a}$ and $5 \mathrm{~d}$ ), suggesting that ROS is implicated, at least in part, in this process. Porphyrins can generate ROS by either electron or hydride transfer or by energy transfer, generating $\mathrm{O}_{2}^{\bullet-}$ and singlet oxygen $\left({ }^{1} \mathrm{O}_{2}\right)^{37,38}$, among other species. Superoxide can be further dismutated to $\mathrm{H}_{2} \mathrm{O}_{2}$, which can react with $\mathrm{Fe}^{2+}$ to generate ${ }^{\bullet} \mathrm{OH}$ ultimately. Our results show that tellurite exposure leads to ${ }^{\bullet} \mathrm{OH}$ generation and iron depletion, and that DP increases tellurite resistance (Fig. 5a-c), supporting a role for ${ }^{\bullet} \mathrm{OH}$ in tellurite toxicity. Interestingly, E. coli does not have dedicated enzymes able to detoxify ${ }^{\bullet} \mathrm{OH}$ or ${ }^{1} \mathrm{O}_{2}$, and metabolites such as reduced thiols have been implicated in the protection against these $\operatorname{ROS}^{38}$. As tellurite depletes the intracellular pool of reduced thiols (Supplementary Fig. 3e), this

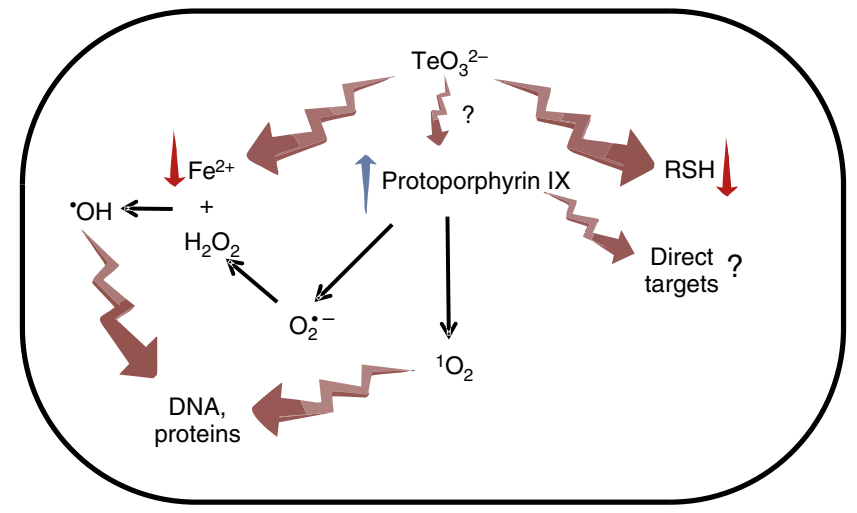

Figure 6 | Schematic model for tellurite and proto IX toxicity in E. coli. Once inside the cell, tellurite depletes the pool of reduced thiols (RSHs) and targets iron-sulfur clusters. As a response, E. coli decreases the pool of intracellular iron, substrate for the enzyme ferrochetalase together with proto IX, causing its accumulation. Alternatively, tellurite could directly inactivate enzymes of the last two steps of heme biosynthesis (?), resulting in protoporphyrin IX accumulation. Protoporphyrin IX directly targets unidentified cellular targets (?) and also acts as a substrate for the production of superoxide $\left(\mathrm{O}_{2}^{\mathbf{}^{-}}\right)$and/or singlet oxygen $\left({ }^{1} \mathrm{O}_{2}\right)$ by electron or energy transfer, respectively. The $\mathrm{O}_{2}^{--}$is dismutated to hydrogen peroxide $\left(\mathrm{H}_{2} \mathrm{O}_{2}\right)$, which reacts with the free $\mathrm{Fe}^{2+}$ (Fenton reaction) derived from the direct action of tellurite, generating the highly toxic hydroxyl radical $\left(\bullet^{\bullet} \mathrm{OH}\right)$. As E. coli does not encode enzymes for the detoxification of ${ }^{\bullet} \mathrm{OH}$ or ${ }^{1} \mathrm{O}_{2}$ and the defensive RSHs are depleted, these ROS, together with protoporphyrin IX, target intracellular macromolecules, causing cell death. $\mathrm{TeO}_{3}^{2}{ }^{-}$: tellurite.

could explain why increased levels of proto IX are toxic only in the presence of tellurite, and why ALA potentiates its toxicity (Fig. 3a,b). Our results support a model where under aerobic conditions, proto IX is a major source of ${ }^{\bullet} \mathrm{OH}$, and most likely also of ${ }^{1} \mathrm{O}_{2}$, which in the absence of any additional stress can be tolerated by the cell. However, on tellurite exposure, the intracellular levels of iron are altered and the pool of reduced thiols is depleted, fuelling the production of ${ }^{\bullet} \mathrm{OH}$ and ${ }^{1} \mathrm{O}_{2}$ and depleting the defense systems against them, leading to cell death. In turn, the increased tellurite resistance observed in strains EM2, $\Delta h e m N$ and $\Delta h e m F$ results by limiting proto IX levels, the source of ${ }^{\bullet} \mathrm{OH}$ and ${ }^{1} \mathrm{O}_{2}$ (Fig. 6).

Little is known about the toxicity of porphyrins and tellurite under anaerobic conditions; however, our results show that ALA potentiates tellurite toxicity under this condition (Fig. 5d), suggesting that porphyrins, and most likely proto IX, could also be toxic. An alternative explanation for this effect could be a direct oxidation of ALA by tellurite. Under aerobic conditions and after enolization, ALA is oxidized by molecular oxygen with the subsequent production of superoxide and hydroxyl radical ${ }^{42}$ It is possible that under both aerobic and anaerobic conditions, tellurite instead of oxygen could oxidize ALA, which could explain the observed cell death under anaerobic conditions (Fig. 5d). ${ }^{1} \mathrm{H}$ nuclear magnetic resonance (NMR) analysis suggested that this reaction is possible, although after long periods (Supplementary Fig. 8). Whether this reaction occurs at a faster rate inside cells that could explain the rapid decrease in viability that is observed (Fig. 4a) remains to be addressed.

In E. coli, tellurite exposure caused the accumulation of intermediates in heme biosynthesis (Fig. 3a), mainly proto IX. Several non-exclusive mechanisms could explain this effect. Tellurite exposure caused a rapid decrease of intracellular iron (Fig. 5c), most likely as a response to prevent ${ }^{\bullet} \mathrm{OH}$ generation (Fig. 5a). However, this defense mechanism could be problematic 
to cope with heme biosynthesis, as iron, together with proto IX, are substrates for the last enzyme in heme biosynthesis, ferrochetalase. The result would be an accumulation of proto IX due to decreased iron availability. In turn, this could lead to further ${ }^{\bullet} \mathrm{OH}$ generation or direct damage by proto IX. In E. coli, $\mathrm{H}_{2} \mathrm{O}_{2}$ exposure also causes proto IX accumulation, and the cell responds by increasing the expression of hemF to ensure heme availability for the enzyme catalase ${ }^{34}$. However, proteomic and transcriptomic studies showed that no genes of the heme biosynthetic pathway are differentially expressed after tellurite exposure ${ }^{19,20}$, strongly suggesting that limiting iron levels causes proto IX accumulation. Further supporting the view that tellurite and $\mathrm{H}_{2} \mathrm{O}_{2}$ have different resistance and toxicity mechanisms in E. coli, DP increased tellurite resistance (Fig. 5c), while it sensitizes the cell to $\mathrm{H}_{2} \mathrm{O}_{2}$ (ref. 34). Another explanation for the increased levels of proto IX after tellurite exposure could be a direct damage to enzymes of the pathway. Previous studies showed that the heavy metals copper, silver, mercury and lead inhibit the activity of purified ferrochetalase and coproporphyrin III oxidase from Rhodopseudomonas sphaeroides and Chromatium D, respectively ${ }^{43,44}$, which could lead to increased levels of proto IX. Whether tellurite exerts its toxicity through a similar mechanism in E. coli remains to be addressed.

In recent years, the global rise of antibiotic resistance has led to an urgent need to (re)discover novel treatments to control pathogens. In the past decade, PDT using heme intermediates, including ALA, has re-emerged as an alternative as it has shown to be effective in controlling cancerous cells, viruses, fungi, parasites and both Gram-positive and -negative bacteria ${ }^{24}$. One major advantage for the use of ALA combined with PDT is that ALA is a prodrug and is toxic only intracellularly where it is metabolized to proto IX, which in turn acts as a source of ROS when photoactivated ${ }^{24}$. However, one major limitation in the use of ALA and other heme derivatives is that, in some cases, millimolar doses are required to cause an effect ${ }^{45,46}$. In this context, the results presented herein could represent a step ahead in this area, as tellurite and ALA potentiate their toxicity and only micromolar doses of both compounds are enough to kill E. coli (Fig. 4a,d,e). Additionally, as proto IX further accumulates in cells grown with ALA and tellurite (Fig. 4b), their combined use could improve the efficacy of PDT by providing a greater source of free radicals to kill microorganisms. Supporting this hypothesis, ALA and tellurite were more efficient at killing E. coli in aerobiosis (Fig. 4a), which could be explained by increased ROS generation under these conditions. It is reasonable to speculate that the combined use of ALA and tellurite could be effective in killing other organisms besides E. coli. Preliminary experiments showed that under the same conditions used in this study, ALA potentiated tellurite toxicity and strongly inhibited the growth of Salmonella Typhimurium and S. Typhi, and to a lesser extent of Klebsiella pneumoniae, however, had little or no effect over Pseudomonas aeruginosa and a clinical strain of E. coli (Supplementary Fig. 9). This was somehow expectable, as higher concentrations of ALA and/or tellurite might be required to achieve a similar effect in different organisms, which must be determined experimentally. Furthermore, designing efficient delivery methods of ALA/tellurite adducts or synthesizing alloys between these two compounds could further improve this process and expand its use to other fields.

\footnotetext{
Methods

Bacterial strains and growth conditions. All strains are derivatives of $E$. coli K-12 strain BW25113 (National Institute of Genetics, Microbial Genetics Laboratory, NBRP, Japan). Strains EM40, EM41 and EM2 were isolated after 3, 13 or 26 aerobic serial passages (one serial passage $=24 \mathrm{~h}$ ) of strain BW25113 in LB medium with increasing tellurite concentrations up to $400 \mu \mathrm{g} \mathrm{ml}^{-1}$ (Fig. 1a). Strains were
}

isolated by single-colony purification. The genomes of strains BW25113, EM41, EM42 and EM2, as well as the identification of mutations in each strain were determined by Macrogen Inc. (South Korea) using the Illumina HiSeq 2000 platform. Single-nucleotide polymorphisms in comparison with the reference genome of E. coli strain BW25113 (GenBank: CP009273.1) are deposited at doi: 10.6084/ m9.figshare.4747402 (Supplementary Data 2). Strains $\Delta$ hem $N$ and $\Delta h e m F$ are derivatives of the Keio collection ${ }^{33}$. Strain EM42 was generated by replacing the wild-type allele with hemA[31E] in strain BW25113 (EM2 DNA was used as a template for PCR reactions). Strains EM43, EM44, EM 64, EM70 and EM71 were generated by replacing the mutant gene with the corresponding wild-type allele in strain EM2 (BW25113 DNA was used as a template for PCR reactions). To achieve this, the different genes were amplified using the primers listed in Supplementary Table 2 and denoted as GIBSON_followed by the name of the gene to be amplified. The resultant PCR products were cloned into plasmid pKD3 (ref. 47) using Gibson assembly ${ }^{48}$. Plasmid pKD3 was amplified using primers Gibson_pKD3F and Gibson_pKD3R. The resulting PCR product was used in all reactions as the vector backbone. The resulting plasmids were used as templates to amplify the gene of interest and the selection marker (caf) using the primers listed in Supplementary Table 2 (named 'wanner_' followed by the name of the gene to be amplified). All strains were constructed using $\lambda$ red recombination ${ }^{47}$ of the corresponding PCR products into strains EM2 or BW25113. Incorporation of the desired mutation in the chromosome was confirmed by PCR and DNA sequencing.

Growth curves were conducted in M9 medium ${ }^{49}$ plus $0.2 \%$ glucose containing or not potassium tellurite (Sigma-Aldrich), $\mathrm{H}_{2} \mathrm{O}_{2}$ (Merck) supplemented or not with $25 \mu \mathrm{g} \mathrm{ml}^{-1}$ ALA (Sigma-Aldrich), as indicated in each figure, in 48-well plates (Nunc). Growth was started by adding $10 \mu \mathrm{l}$ of an overnight culture of each strain grown in M9 medium into a well containing $990 \mu \mathrm{l}$ (1:100 dilution) of the medium indicated in each figure. Growth was conducted at $37^{\circ} \mathrm{C}$ in a Tecan INFINITE M200 Pro with constant shaking at 141.9 r.p.m. and the optical density (OD) at $600 \mathrm{~nm}$ was monitored every $15 \mathrm{~min}$ for $24 \mathrm{~h}$. Since tellurite is reduced to tellurium inside cells (Supplementary Fig. 10), OD $600 \mathrm{~nm}$ might be overestimated in some cases, impeding the accurate calculation of specific growth rates and half-maximal inhibitory concentration values.

For cell viability experiments, overnight cultures grown in M9 medium were diluted (1:100) into $250 \mathrm{ml}$ flasks containing $25 \mathrm{ml}$ of M9 medium with or without tellurite, $\mathrm{H}_{2} \mathrm{O}_{2}, 1 \mathrm{mM} 2,2^{\prime}$-DP (Sigma-Aldrich), supplemented or not with $25 \mathrm{\mu g} \mathrm{ml}^{-1}$ ALA (as indicated in each figure) and CFU were determined at the indicated time points. Cultures were grown at $37^{\circ} \mathrm{C}$ with an agitation of 250 r.p.m. For anaerobic experiments (Fig. 5d), cells were grown inside an anaerobic chamber (Coy Laboratory Products) at $37^{\circ} \mathrm{C}$ with an agitation of 125 r.p.m. Growth was started as described above but under anaerobic conditions. All solutions were equilibrated inside the anaerobic chamber for at least 1 week before conducting the experiments.

Chemical genomic profiling of tellurite with $\mathbf{E}$. coli. Pooled competition experiments of the barcoded $E$. coli deletion collection ${ }^{30}$ were grown in the presence of potassium tellurite $\left(0.63 \mu \mathrm{g} \mathrm{ml}^{-1}\right)$ or a DMSO (solvent). Each pooled competition was grown in $200 \mu \mathrm{l}$ cultures $(n=3)$ at $37^{\circ} \mathrm{C}$ for $24 \mathrm{~h}$ in a TECAN M1000. After $24 \mathrm{~h}$, genomic DNA was extracted using the Invitrogen PureLink 96-well genomic DNA Extraction Kit (Cat No. K1821-04 A). Strain specific barcodes from each culture were amplified using indexed primers designed for multiplexed Illumina sequencing. The forward primer contained the Illumina specific P5 sequence, a 10 bp index tag (x's), and the 19 bp E. coli deletion collection common priming site: $5^{\prime}$-AATGATACGGCGACCACCGAGATCTACACTCTTT CCCTACACGACGCTCTTCCGATCT $x x x x x x x x x x A A T C T T C G G T A G T C C A$ GCG- $3^{\prime}$. The reverse primer contained the Illumina-specific P7 sequence and $20 \mathrm{bp}$ E. coli common priming site: $5^{\prime}$-CAAGCAGAAGACGGCATACGAGCTCTTCC GATCTTGTAGGCTGGAGCTGCTTCG-3'. Barcodes were amplified by PCR, pooled, gel purified and quantified by quantitative PCR as described ${ }^{50}$. For barcode sequencing, samples were run on an Illumina HiSeq2500 in rapid run mode for 50 cycles at a loading concentration of $15 \mathrm{pM}$. The resulting Fastq file was used for analysis of sensitive and resistant mutants. We compared normalized counts to a solvent (DMSO) control to identify compound specific responses among gene deletion mutants (chemical genetic interaction score). Sequence data was processed using BEANcounter (https://github.com/csbio/BEAN-counter) and EdgeR ${ }^{51}$.

Extraction and measurement of intracellular ALA and heme. Overnight cultures of the indicated strains grown in M9 medium were diluted (1:100) into $500 \mathrm{ml}$ flasks containing $150 \mathrm{ml} \mathrm{M} 9$ medium supplemented or not with $25 \mu \mathrm{g} \mathrm{ml}^{-1}$ ALA. Cultures were grown at $37^{\circ} \mathrm{C}$ to an OD $600 \mathrm{~nm}$ of 0.4 and intracellular ALA was extracted from a $100 \mathrm{ml}$ aliquot as described previously ${ }^{52}$ and quantified using Ehrlich's reagent (Sigma-Aldrich) by following absorbance at $553 \mathrm{~nm}$. Intracellular heme was extracted from $1 \mathrm{ml}$ of the same cultures as described previously ${ }^{53}$ and fluorescence was determined in a Tecan INFINITE M200 Pro with an excitation wavelength of $400 \mathrm{~nm}$ and emission wavelengths of 610 and $660 \mathrm{~nm}$. For ALA and heme, values were normalized by the OD $600 \mathrm{~nm}$ of the culture.

Porphyrin extraction and quantification. Overnight cultures of the indicated strains grown in M9 medium were diluted (1:100) into 21 of M9 medium 
supplemented or not with $25 \mu \mathrm{g} \mathrm{ml}^{-1}$ ALA and grown aerobically at $37^{\circ} \mathrm{C}$ to an OD $600 \mathrm{~nm}$ of 0.4 . At this point, $25 \mu \mathrm{g} \mathrm{ml}^{-1}$ tellurite was added to the cultures and porphyrins were extracted from 11 (cells grown in M9 medium) or $400 \mathrm{ml}$ (cells grown in M9 medium with ALA) of the cultures at the indicated time points as described previously ${ }^{54}$

Porphyrins were analysed and quantified as described by Tatsumi and Wachi ${ }^{45}$ using a 1,200 series high-performance liquid chromatography (HPLC) system (Agilent Technologies) including a degasser (G1322A), a quaternary pump (G1311A), a variable wavelength detector (G1314B) and a manual sampler. The injection volume was $20 \mu \mathrm{l}$. The LC separation was performed on an Agilent Zorbax Eclipse XDB-C18 column $\left(4.6 \times 150 \mathrm{~mm}^{2}, 5 \mu \mathrm{m}\right)$ eluted for $45 \mathrm{~min}$ at a flow rate of $1 \mathrm{ml} \mathrm{min}^{-1}$ with a linear gradient from $50 \%$ solvent A $(10 \%$ acetonitrile in $1 \mathrm{M}$ ammonium acetate; $\mathrm{pH} 5.1$ ), $50 \%$ solvent $\mathrm{B}$ (methanol-acetic acid $10: 1, \mathrm{vv}^{-1}$ ) to $100 \%$ solvent $\mathrm{B}$, followed by elution with $100 \%$ solvent $\mathrm{B}$ for $5 \mathrm{~min}$. The porphyrin composition of each sample was analysed following the absorbance at $300 \mathrm{~nm}$. Commercially available standards of coproporphyrin III and proto IX (Frontier Scientific) were used to identify and quantify each compound from the different samples. Values were normalized by $\mathrm{mg}$ of protein as determined by the Bradford method ${ }^{55}$.

Biochemical determinations. For all biochemical determinations, overnight cultures of the indicated strains grown in M9 medium were inoculated into $250 \mathrm{ml}$ flasks containing $125 \mathrm{ml}$ of M9 medium supplemented or not with $25 \mu \mathrm{g} \mathrm{ml}^{-1}$ ALA (indicated in each figure). Cultures were grown aerobically at $37^{\circ} \mathrm{C}$ with an agitation of 250 r.p.m. to an OD $600 \mathrm{~nm}$ of 0.4. At this time, cells were treated with either tellurite or $\mathrm{H}_{2} \mathrm{O}_{2}$, as indicated in each figure. At each of the indicated time points, a $25 \mathrm{ml}$ aliquot was centrifuged at $8,000 \mathrm{~g}$ at $4{ }^{\circ} \mathrm{C}$ for $5 \mathrm{~min}$ and the cell pellet was washed two times with $5 \mathrm{ml}$ of $50 \mathrm{mM}$ Tris- $\mathrm{HCl}$ (pH 7.4). The cell pellet was suspended in $500 \mu \mathrm{l}$ of $50 \mathrm{mM}$ Tris- $\mathrm{HCl}(\mathrm{pH}$ 7.4) and cells were disrupted by sonication. For catalase activity, cell pellet was suspended in $50 \mathrm{mM}$ phosphate saline buffer ( $\mathrm{pH}$ 7.4). Cell-free extracts recovered after centrifugation at $14,000 \mathrm{~g}$ at $4{ }^{\circ} \mathrm{C}$ for $20 \mathrm{~min}$ were used to determine the different activities. For all cases, OD was monitored using a Tecan INFINITE M200 Pro. Values were normalized by $\mathrm{mg}$ of protein as determined by the Bradford method ${ }^{55}$.

Catalase activity. Catalase activity was determined in $200 \mu \mathrm{l}$ reactions using $10 \mu \mathrm{l}$ of cell-free extract as described previously ${ }^{56}$ by following $\mathrm{H}_{2} \mathrm{O}_{2}$ consumption at $240 \mathrm{~nm}$. Catalase activity was defined as nmoles $\mathrm{H}_{2} \mathrm{O}_{2}$ consumed $\mathrm{min}^{-1} \mathrm{mg}$ protein $^{-1}$.

Superoxide dismutase activity. Activity was determined in $200 \mu \mathrm{l}$ reactions using $10 \mu \mathrm{l}$ of cell-free extract in buffer $50 \mathrm{mM}$ Tris- $\mathrm{HCl}$ (pH 7.4), $1 \mathrm{mM}$ EDTA with $25 \mu \mathrm{M}$ nitroblue tetrazolium (Sigma-Aldrich), $50 \mu \mathrm{M}$ xanthine and $0.25 \mu \mathrm{g} \mu \mathrm{l}^{-1}$ xanthine oxidase (Sigma-Aldrich). Superoxide dismutase activity activity was monitored following NTB oxidation at $412 \mathrm{~nm}$ as described previously ${ }^{56}$. One unit (U) of superoxide dismutase activity activity was defined as the amount of enzyme required to inhibit in $50 \%$ NBT oxidation $\mathrm{min}^{-1} \mathrm{mg}$ protein ${ }^{-1}$.

TR activity. TR activity was determined in $200 \mu \mathrm{l}$ reactions using $10 \mu \mathrm{l}$ of cell free extract in buffer $50 \mathrm{mM}$ Tris- $\mathrm{HCl}(\mathrm{pH} 7.4), 1 \mathrm{mM}$ tellurite, $1 \mathrm{mM} \mathrm{NAD}(\mathrm{P}) \mathrm{H}$ (Sigma-Aldrich) and $1 \mathrm{mM} \beta$-mercaptoethanol (Sigma-Aldrich). TR activity was monitored following the appearance of tellurium at $500 \mathrm{~nm}$ as described previously ${ }^{57}$. One unit (U) of TR activity was defined as the amount of enzyme required to increase the OD $500 \mathrm{~nm}$ in $0.001 \mathrm{~min}^{-1} \mathrm{mg}_{\text {protein }}{ }^{-1}$.

Total thiol content. Total thiol content was quantified using Ellman's reagent $5,5^{\prime}$-dithiobis-(2-nitrobenzoic acid) (Sigma-Aldrich) as described previously ${ }^{58}$ by following $\mathrm{TNB}^{-2}$ generation at OD $412 \mathrm{~nm}$. Values were normalized by $\mathrm{mg}$ protein.

Inductively coupled plasma-optical emission spectrometry. Inductively coupled plasma-optical emission spectrometry (ICP-OES) analysis was performed using a Perkin-Elmer optima 2000 DV apparatus in the axial viewing mode. The wavelength corresponding to tellurium was $214,281 \mathrm{~nm}$. Calibration curves for tellurium and iron (in $2 \%$ ultrapure $\mathrm{HNO}_{3}$ ) were constructed. Briefly, overnight cultures of the indicated strains grown in M9 medium were inoculated (1:100 dilution) into $250 \mathrm{ml}$ flasks containing $125 \mathrm{ml}$ of $\mathrm{M} 9$ medium. Cultures were grown aerobically at $37^{\circ} \mathrm{C}$ with an agitation of 250 r.p.m. to an OD $600 \mathrm{~nm}$ of 0.4 . At this time, cells were treated with tellurite. At the indicated time points, a $25 \mathrm{ml}$ aliquot was centrifuged at $8,000 \mathrm{~g}$ at $4^{\circ} \mathrm{C}$ for $5 \mathrm{~min}$ and the cell pellet was washed twice with $5 \mathrm{ml}$ of $50 \mathrm{mM}$ Tris- $\mathrm{HCl}$ (pH 7.4). The cell pellet was weighed, and suspended in $8 \mathrm{ml}$ of $2 \% \mathrm{HNO}_{3}$ and subjected to ICP-OES analyses. Values were normalized by the weight of the cell pellet.

${ }^{1} \mathbf{H}$ NMR analysis. The analysis was performed on a Bruker Avance $400 \mathrm{MHz}$ spectrometer $\left(400.133 \mathrm{MHz}\right.$ for $\left.{ }^{1} \mathrm{H}\right)$ equipped with a 5 -mm multinuclear broadband dual probe head incorporating a $\mathrm{z}$-gradient coil as described previously ${ }^{59}$.
All the measurements were carried out in M9 medium with $5 \mathrm{mg}$ ALA and with or without $5 \mathrm{mg}$ tellurite. Chemical shifts were calibrated with respect to the solvent signal ( $\delta=7.26$ p.p.m. for proton residual solvent) and referenced to TMS.

Hydroxyl radical generation. To determine hydroxyl radical generation, overnight cultures of strain BW25113 grown in M9 medium were inoculated (1:100 dilution) into $125 \mathrm{ml}$ flasks containing $25 \mathrm{ml}$ of $\mathrm{M} 9$ medium supplemented or not with $25 \mu \mathrm{g} \mathrm{ml}^{-1}$ ALA (indicated in each figure). Cultures were grown aerobically at $37^{\circ} \mathrm{C}$ with an agitation of 250 r.p.m. to an OD $600 \mathrm{~nm}$ of 0.4 . At this time, the probe hydroxyphenyl fluorescein was added to the culture $(5 \mu \mathrm{M}$ final concentration) and cells were grown for $20 \mathrm{~min}$. Cells were washed twice with $25 \mathrm{ml}$ of $50 \mathrm{mM}$ Tris- $\mathrm{HCl} \mathrm{pH} 7.4$ to remove excess probe. Following, the cell pellet was suspended in $25 \mathrm{ml}$ of $\mathrm{M} 9$ medium, and $190 \mu \mathrm{l}$ aliquots were added to individual wells of a 96 well plate. At this point, the growth medium was supplemented with the indicated concentrations of tellurite, $25 \mu \mathrm{g} \mathrm{ml}^{-1}$ ALA or $5 \mathrm{mM}$ thiourea (Merck), and growth was continued in a Tecan INFINITE M200 Pro at $37^{\circ} \mathrm{C}$. Hydroxyl radical production was followed by measuring the fluorescence of the probe hydroxyphenyl fluorescein at the indicated time points with an excitation wavelength of $490 \mathrm{~nm}$ and an emission wavelength of $525 \mathrm{~nm}$. Values were normalized by OD $600 \mathrm{~nm}$.

Data availability. The chemical genomic profiling and single-nucleotide polymorphism data sets (Supplementary Data 1 and 2, respectively) generated during and/or analysed in the current study have been deposited in Figshare with the identifier doi: 10.6084/m9.figshare.4747402 (ref. 60). The authors declare that all other relevant data supporting the findings of this study are available within the paper and its Supplementary Information files, or from the corresponding authors on request.

\section{References}

1. Fleming, A. On the specific antibacterial properties of penicillin and potassium tellurite. Incorporating a method demonstrating some bacterial antagonisms. J. Pathol. Bacteriol. 35, 831-842 (1932).

2. Franke, K. W. \& Moxon, A. L. A comparison of the minimum fatal doses of selenium, tellurium, arsenic and vanadium. J. Pharmacol. Exp. Ther. 58, 454-459 (1936).

3. Taylor, D. E. Bacterial tellurite resistance. Trends Microbiol. 7, 111-115 (1999).

4. Nies, D. Microbial heavy-metal resistance. Appl. Microbiol. Biotechnol. 51, 730-750 (1999).

5. Turner, R. J., Hou, Y., Weiner, J. H. \& Taylor, D. E. The arsenical ATPase efflux pump mediates tellurite resistance. J. Bacteriol. 174, 3092-3094 (1992).

6. Taylor, D. E., Hou, Y., Turner, R. J. \& Weiner, J. H. Location of a potassium tellurite resistance operon (tehAtehB) within the terminus of Escherichia coli K-12. J. Bacteriol. 176, 2740-2742 (1994).

7. Turner, R. J., Weiner, J. H. \& Taylor, D. E. The tellurite resistance determinants teh $A$ tehB and klaA klaB telB have different biochemical requirements. Microbiology 141, 3133-3140 (1995).

8. Goncharoff, P., Saadi, S., Chang, C. H., Saltman, L. H. \& Figurski, D. H. Structural, molecular, and genetic analysis of the kilA operon of broad-hostrange plasmid RK2. J. Bacteriol. 173, 3463-3477 (1991).

9. Walter, E. G., Thomas, C. M., Ibbotson, J. P. \& Taylor, D. E. Transcriptional analysis, translational analysis, and sequence of the KilA-tellurite resistance region of plasmid RK2Ter. J. Bacteriol. 173, 1111-1119 (1991).

10. Kormutakova, R., Klucar, L. \& Turna, J. DNA sequence analysis of the telluriteresistance determinant from clinical strain of Escherichia coli and identification of essential genes. Biometals 13, 135-139 (2000).

11. Chasteen, T. G., Fuentes, D. E., Tantaleán, J. C. \& Vásquez, C. C. Tellurite: history, oxidative stress, and molecular mechanisms of resistance. FEMS Microbiol. Rev. 33, 820-832 (2009).

12. Turner, R. J., Weiner, J. H. \& Taylor, D. E. Neither reduced uptake nor increased efflux is encoded by tellurite resistance determinants expressed in Escherichia coli. Can. J. Microbiol. 41, 92-98 (1995).

13. Turkovicova, L. et al. Proteomic analysis of the TerC interactome: novel links to tellurite resistance and pathogenicity. J. Proteomics 136, 167-173 (2016).

14. Turner, R. J., Aharonowitz, Y., Weiner, J. H. \& Taylor, D. E. Glutathione is a target in tellurite toxicity and is protected by tellurite resistance determinants in Escherichia coli. Can. J. Microbiol. 47, 33-40 (2001).

15. Anaganti, N., Basu, B., Gupta, A., Joseph, D. \& Apte, S. K. Depletion of reduction potential and key energy generation metabolic enzymes underlies tellurite toxicity in Deinococcus radiodurans. Proteomics 15, 89-97 (2015)

16. Tremaroli, V., Fedi, S. \& Zannoni, D. Evidence for a tellurite dependent generation of reactive oxygen species and absence of a tellurite-mediated adaptive response to oxidative stress in cells of Pseudomonas pseudoalcaligenes KF707. Arch. Microbiol. 187, 127-135 (2007).

17. Pérez, J. M. et al. Bacterial toxicity of potassium tellurite: unveiling an ancient enigma. PLoS ONE 2, e211 (2007). 
18. Aradská, J., Ŝmidák, R., Turkoviĉová, L., Turňa, J. \& Lubec, G. Proteomic differences between tellurite-sensitive and tellurite-resistant E. coli. PLOS ONE 8, e78010 (2013).

19. Molina-Quiroz, R. C. et al. Global transcriptomic analysis uncovers a switch to anaerobic metabolism in tellurite-exposed Escherichia coli. Res. Microbiol. 165, 566-570 (2014)

20. Vrionis, H. A., Wang, S., Haslam, B. \& Turner, R. J. Selenite protection of tellurite toxicity toward Escherichia coli. Front. Mol. Biosci. 18, 69 (2015).

21. Tantaleán, J. C. et al. The Geobacillus stearothermophilus $\mathrm{V}$ iscS gene, encoding cysteine desulfurase, confers resistance to potassium tellurite in Escherichia coli K-12. J. Bacteriol. 185, 5831-5837 (2003).

22. Rathgeber, C., Yurkova, N., Stackebrandt, E., Beatty, J. T. \& Yurkov, V. Isolation of tellurite- and selenite-resistant bacteria from hydrothermal vents of the Juan de Fuca Ridge in the Pacific Ocean. Appl. Environ. Microbiol. 68, 4613-4622 (2002).

23. Ollivier, P. R. et al. Volatilization and precipitation of tellurium by aerobic, tellurite-resistant marine microbes. Appl. Environ. Microbiol. 74, 7163-7173 (2008).

24. Harris, F. \& Pierpoint, L. Photodynamic therapy based on 5-aminolevulinic acid and its use as an antimicrobial agent. Med. Res. Rev. 32, 1292-1327 (2012).

25. Arenas, F. A. et al. Isolation, identification and characterization of tellurite hyper-resistant, tellurite-reducing bacteria from Antarctica. Polar Sci. 8, 40-52 (2014).

26. Macomber, L. \& Imlay, J. A. The iron-sulfur clusters of dehydratases are primary intracellular targets of copper toxicity. Proc. Natl Acad. Sci. USA 106, 8344-8349 (2009)

27. Alper, H. \& Stephanopoulos, G. Global transcription machinery engineering a new approach for improving cellular phenotype. Metab. Eng. 9, 258-267 (2007).

28. Jovanovic, G., Weiner, L. \& Model, P. Identification, nucleotide sequence, and characterization of $\mathrm{PspF}$, the transcriptional activator of the Escherichia coli stress-induced psp operon. J. Bacteriol. 178, 1936-1945 (1996).

29. Avissar, Y. J. \& Beale, S. I. Identification of the enzymatic basis for delta-aminolevulinic acid auxotrophy in a hemA mutant of Escherichia coli. J. Bacteriol 171, 2919-2924 (1989)

30. Otsuka, Y. et al. GenoBase: comprehensive resource database of Escherichia coli K-12. Nucleic Acids Res. 43, D606-D617 (2015).

31. Troup, B., Hungerer, C. \& Jahn, D. Cloning and characterization of the Escherichia coli hem $N$ gene encoding the oxygen-independent coproporphyrinogen III oxidase. J. Bacteriol. 177, 3326-3331 (1995).

32. Xu, K., Delling, I. J. \& Elliott, T. The genes required for heme synthesis in Salmonella typhimurium include those encoding alternative functions for aerobic and anaerobic coproporphyrinogen oxidation. J. Bacteriol. 174, 3953-3963 (1992).

33. Baba, T. et al. Construction of Escherichia coli K-12 in-frame, single-gene knockout mutants: the Keio collection. Mol. Syst. Biol. 2, 2006.0008 (2006).

34. Mancini, S. \& Imlay, J. A. The induction of two biosynthetic enzymes helps Escherichia coli sustain heme synthesis and activate catalase during hydrogen peroxide stress. Mol. Microbiol. 96, 744-763 (2015).

35. Elliott, T. Transport of 5-aminolevulinic acid by the dipeptide permease in Salmonella typhimurium. J. Bacteriol. 175, 325-331 (1993).

36. Calderón, I. L. et al. Tellurite-mediated disabling of [4Fe-4S] clusters of Escherichia coli dehydratases. Microbiology 155, 1840-1846 (2009).

37. Miyamoto, K., Nakahigashi, K., Nishimura, K. \& Inokuchi, H. Isolation and characterization of visible light sensitive mutants of Escherichia coli K12. J. Mol. Biol. 219, 393-398 (1991).

38. Vatansever, F. et al. Antimicrobial strategies centered around reactive oxygen species-bactericidal antibiotics, photodynamic therapy, and beyond. FEMS Microbiol. Rev. 37, 955-989 (2013).

39. Martin, J. P. \& Logsdon, N. Oxygen radicals are generated by dye-mediated intracellular photooxidations: a role for superoxide in photodynamic effects. Arch. Biochem. Biophys. 256, 39-49 (1987).

40. Moser, J. et al. V-shaped structure of glutamyl-tRNA reductase, the first enzyme of tRNA-dependent tetrapyrrole biosynthesis. EMBO J. 20, 6583-6590 (2001).

41. Li, G. W., Burkhardt, D., Gross, C. \& Weissman, J. S. Quantifying absolute protein synthesis rates reveals principles underlying allocation of cellular resources. Cell 157, 624-635 (2014).

42. Monteiro, H. P., Abdalla, D. S., Augusto, O. \& Bechara, E. J. Free radical generation during delta-aminolevulinic acid autoxidation: induction by hemoglobin and connections with porphyrinpathies. Arch. Biochem. Biophys. 271, 206-216 (1989).

43. Dailey, H. A. Purification and characterization of membrane-bound ferrochelatase from Rhodopseudomonas sphaeroides. J. Biol. Chem. 257, 14714-14718 (1982).

44. Mori, M. \& Sano, S. Studies on the formation of protoporphyrin IX by anaerobic bacteria. Biochim. Biophys. Acta 264, 252-262 (1972).

45. Tatsumi, R. \& Wachi, M. TolC-dependent exclusion of porphyrins in Escherichia coli. J. Bacteriol. 190, 6228-6233 (2008)
46. Morimoto, K. et al. Photodynamic therapy using systemic administration of 5-aminolevulinic acid and a 410-nm wavelength light-emitting diode for methicillin-resistant Staphylococcus aureus-infected ulcers in mice. PLoS ONE 9, e105173 (2014).

47. Datsenko, K. A. \& Wanner, B. L. One-step inactivation of chromosomal genes in Escherichia coli K-12 using PCR products. Proc. Natl Acad. Sci. USA 97, 6640-6645 (2000).

48. Gibson, D. G. et al. Enzymatic assembly of DNA molecules up to several hundred kilobases. Nat. Methods 6, 343-345 (2009).

49. Miller, J. H. Experiments in Molecular Genetics (Cold Spring Harbor Laboratory Press, 1972).

50. Piotrowski, J. S. et al. Chemical genomic profiling via barcode sequencing to predict compound mode of action. Methods Mol. Biol. 1263, 299-318 (2015).

51. Robinson, M. D., McCarthy, D. J. \& Smyth, G. K. edgeR: a Bioconductor package for differential expression analysis of digital gene expression data. Bioinformatics 26, 139-140 (2010).

52. Kanjo, N., Nakahigashi, K., Oeda, K. \& Inokuchi, H. Isolation and characterization of a cDNA from soybean and its homolog from Escherichia coli, which both complement the light sensitivity of Escherichia coli hemH mutant strain VS101. Genes Genet. Syst. 76, 327-334 (2001).

53. Morrison, G. R. Fluorimetric microdetermination of heme protein. Anal. Chem 37, 1124-1126 (1965).

54. Nakayashiki, T. \& Inokuchi, H. Effects of starvation for heme on the synthesis of porphyrins in Escherichia coli. Mol. Gen. Genet. 255, 376-381 (1997).

55. Bradford, M. M. A rapid and sensitive method for the quantitation of microgram quantities of protein utilizing the principle of protein-dye binding Anal. Biochem. 72, 248-254 (1976).

56. Jakubowski, W., Biliński, T. \& Bartosz, G. Oxidative stress during aging of stationary cultures of the yeast Saccharomyces cerevisiae. Free Radic. Biol. Med. 28, 659-664 (2000).

57. Calderón, I. L. et al. Catalases are $\mathrm{NAD}(\mathrm{P}) \mathrm{H}$-dependent tellurite reductases. PLOS ONE 1, e70 (2006).

58. Turner, R. J., Weiner, J. H. \& Taylor, D. E. Tellurite-mediated thiol oxidation in Escherichia coli. Microbiology 145, 2549-2557 (1999).

59. Jara, D. H. et al. Spectroscopic evidence in solid and solution of a discrete copper(I) tetrahedral complex dimer supported by supramolecular interactions. Eur. J. Inorg. Chem. 10, 1579-1583 (2012).

60. Morales, E. H. et al. Supplementary datasets manuscript 'Accumulation of heme biosynthetic intermediates contributes to the antibacterial action of the metalloid tellurite'. figshare. doi:10.6084/m9.figshare.4747402 (2017).

\section{Acknowledgements}

We thank Dr Juan Guerrero for advice and assistance in ${ }^{1} \mathrm{H}$ NMR analysis. E.H.M. is supported by grant 'Fondecyt Postdoctorado 3150004', CONICYT, Chile. J.P.S. is supported by National Institutes of Health Grants 1R01HG005084. C.L.M. and J.N. are supported by National Institutes of Health Grants 1R01HG005084, 1R01GM104975 and R01HG005853 and National Science Foundation Grant DBI 0953881. S.W.S. is supported by an NSF Graduate Research Fellowship (00039202), an NIH Biotechnology training grant (T32GM008347). F.A.A. is supported by grant 'Fondecyt de Iniciación en la Investigación 11140334', CONICYT, Chile. H.M. is supported by grants JSPS Kakenhi 25250028, 16H02485 and MEXT Kakenhi 25108716, Grant-in-Aid for Scientific Research from the Ministry of Education, Culture, Sports, Science and Technology of Japan. C.C.V. is supported by grants 'Fondecyt Regular 1130362 and 1160051 ', CONICYT, Chile.

\section{Author contributions}

E.H.M. designed and performed all experiments, analysed the data, wrote the paper, provided reagents and publication fee (grant 'Fondecyt Postdoctorado 3150004', CONICYT, Chile). C.A.P. performed all experiments and contributed with partial data analysis. R.L. performed biochemical determinations and strain construction. C.M.M.-V assisted in directed evolution experiments and performed biochemical determinations. F.A.C. performed biochemical determinations. C.L.M. processed and analysed data from chemical genomic experiments. J.N. processed and analysed data from chemical genomic experiments. S.W.S. processed and analysed data from chemical genomic experiments. F.A.A. performed biochemical determinations. J.S.P. performed, processed and analysed data from chemical genomic experiments, wrote the paper and provided reagents. H.M provided the E. coli barcoded deletion collection, edited the paper, and publication fee. C.C.V. designed experiments, wrote the paper and provided reagents.

\section{Additional information}

Supplementary Information accompanies this paper at http://www.nature.com/ naturecommunications

Competing interests: The authors declare no competing financial interests. 
Reprints and permission information is available online at http://npg.nature.com/ reprintsandpermissions/

How to cite this article: Morales, E. H. et al. Accumulation of heme biosynthetic intermediates contributes to the antibacterial action of the metalloid tellurite. Nat. Commun. 8, 15230 doi: 10.1038/ncomms15230 (2017).

Publisher's note: Springer Nature remains neutral with regard to jurisdictional claims in published maps and institutional affiliations. (c) (i) This work is licensed under a Creative Commons Attribution 4.0 International License. The images or other third party material in this article are included in the article's Creative Commons license, unless indicated otherwise in the credit line; if the material is not included under the Creative Commons license, users will need to obtain permission from the license holder to reproduce the material. To view a copy of this license, visit http://creativecommons.org/licenses/by/4.0/

(C) The Author(s) 2017 